Images: Penile necrotic ulcer after prostatic artery embolization

\title{
Images - Penile necrotic ulcer following prostatic artery embolization: A rare complication
}

Félix Couture $^{1}$; François Belzile ${ }^{2}$; Maxime Noël-Lamy²; Yves Ponsot ${ }^{1}$; Le Mai Tu ${ }^{1}$

${ }^{1}$ Department of Surgery, Division of Urology, Centre Hospitalier Universitaire de Sherbrooke, Sherbrooke, QC, Canada; ${ }^{2}$ Department of Radiology, Division of Interventional Radiology, Centre Hospitalier Universitaire de Sherbrooke, Sherbrooke, QC, Canada

Cite as: Can Urol Assoc J 2020 February 4; Epub ahead of print. http://dx.doi.org/10.5489/cuaj.6239

Published online February 4, 2020

$* * *$

\section{Introduction}

Prostatic artery embolization (PAE) has been shown to be a safe and efficacious technique in treating patients with lower urinary tract symptoms (LUTS) secondary to benign prostatic hyperplasia (BPH). (1) PAE is thought to work through degeneration and necrosis of prostatic cells, leading to fibrosis and decrease in overall prostate volume. (2) While selective embolization is usually always performed, non-target embolization may occur, as intravascular particles may reflux into adjacent penile, vesical, or rectal arteries. Ischemia and ulceration of these structures has been reported in most case series and trials, usually manifesting as selflimited lesions and hematuria, hematospermia, or hematochezia. (3) Serious complications secondary to non-target embolization remain rare. We present here a rare case of focal penile necrotic ulceration secondary to non-target embolization during PAE. We provide clinical images of the lesion, as well as procedural radiological images. We also review and discuss the current literature on PAE and its potential complications.

\section{Case report}

We report the case of a 75-year-old male followed by our urology service for a complex, refractory uretral stricture, as well as BPH. He underwent multiple uretral dilatation and uretroplasty, until a cystoscopy revealed significant obstructive BPH, likely responsible for residual LUTS. After discussion, the patient was referred to the interventional radiology department at our instution for PAE.

During the procedure, the left prostatic artery was injected selectively, showing prostatic enhancement with a patent shunt to the left dorsal penile artery. (Figure 1A) This shunt was protected and embolized with a 3-mm detachable intravascular coil. (Figure 1B) The left prostatic artery was safely embolized to stasis with $400 \mu \mathrm{m}$ microparticules. The right prostatic artery was then selected, with a similar shunt to the dorsal penile artery being present. On this side, the penile 
shunt was too small to be secured and selectively embolized with coiling material. (Figure 1C) Arterial flow within the shunt eventually directed itself preferentially toward the prostatic artery, and not toward the penile branch, and embolization was therefore performed with care. The embolization was completed to stasis, and the patient was discharged the same day without any immediate complication.

The patient presented to the emergency department 10 days following the procedure with a painful, dark lesion located on the penile glans that had appeared one week after embolization. Physical examination revealed a necrotic, 1-cm ulcer surrounded by a zone of erythema just adjacent to the right side of the uretral meatus. (Figure 2A) Post-void residual volume was also measured at $350 \mathrm{cc}$, and the patient was discharged with a Foley catheter and analgesia.

Additionally, cystoscopy was performed to rule out any other ulceration or necrosis of the lower urinary tract. Trial of void was successful about 2 weeks after the procedure, and the Foley catheter was removed. At a follow-up visit 3 weeks after the procedure, the penile lesion had healed by about $50 \%$, and had completely resolved after about 5 weeks, without any additional systemic or topical treatment. (Figure $2 B$ )

\section{Discussion}

PAE is a novel endovascular technique used in the treatment of patients with LUTS due to BPH. PAE represents an effective and interesting alternative, especially for patients unfit for TURP, or refusing surgery. During PAE, small particles are injected to occlude bilateral prostatic arteries distally, leading to ischemia and shrinkage of prostatic tissue. (1) While it is considered a safe procedure associated with less overall complications than TURP, (4) PAE requires a high level of expertise, and should be performed by well-trained interventional radiologists to minimize complications. (1) Common post-procedural complications and side effects include pelvic, urethral, and perineal pain, dysuria, urinary tract infection, and hematuria. Gastrointestinal symptoms have also been reported (e.g. diarrhea, rectorrhagia, nausea). (3) Self-resolving AUR is also a well-known complication of this procedure, occurring in about $2.5 \%$ of most case series, (3) and is thought to be due to urethral compression from transient post-procedural inflammatory prostatic edema. (5)

Because of the large variation in prostatic artery anatomy and potential bowel, bladder and penile anastomoses, identification and selective embolization of prostatic vessels can be challenging. (1) Non-target embolization can lead to ischemia, inflammation, and ulceration of affected tissues. Ischemic proctitis, bladder wall infarction, vesical seminitis, and penile ulceration have all been reported, almost all of which were self-resolving, and seldom required surgical intervention. (3) Post-PAE penile ischemic ulceration has usually been managed with simple outpatient analgesia in available studies. (1) 


\section{Conclusion}

We reported and discussed a case of iatrogenic focal penile necrotic ulceration, a rare complication of PAE. We provided clinical and radiological images, and discussed the current state of the literature on PAE and its potential complications. This report should stress the importance of discussing the risk of non-target embolization with patients and staying aware of this risk on follow-up after this procedure.

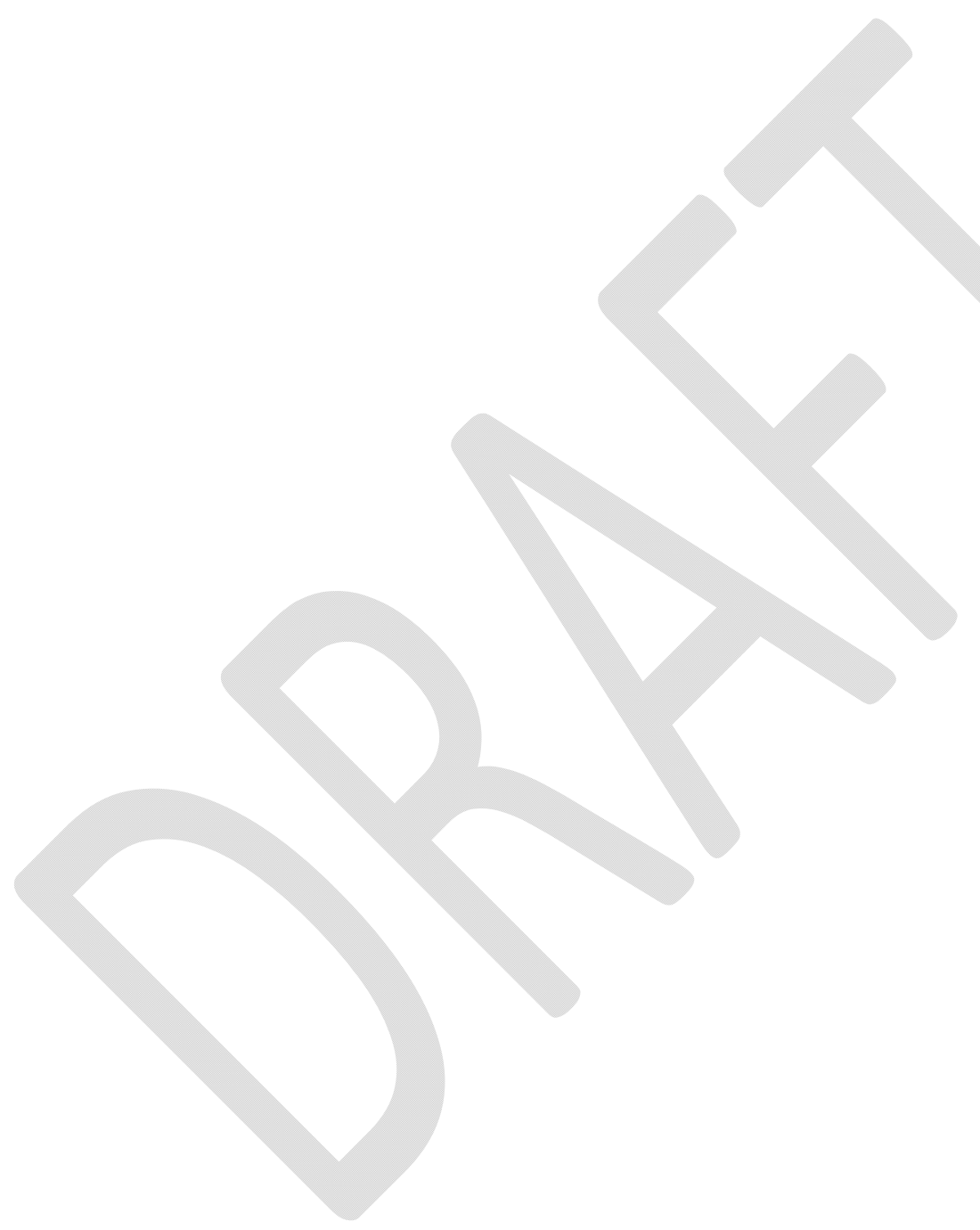


Images: Penile necrotic ulcer after prostatic artery embolization

\section{References}

1. Ray AF, Powell J, Speakman MJ, Longford NT, DasGupta R, Bryant T, et al. Efficacy and safety of prostate artery embolization for benign prostatic hyperplasia: an observational study and propensity-matched comparison with transurethral resection of the prostate (the UK-ROPE study). BJU Int. 2018;122(2):270-82.

2. Chin P. Prostatic artery embolization: adding to the arsenal against the hapless prostate. BJU Int. 2019;123(6):911-2.

3. Moreira AM, de Assis AM, Carnevale FC, Antunes AA, Srougi M, Cerri GG. A Review of Adverse Events Related to Prostatic Artery Embolization for Treatment of Bladder Outlet Obstruction Due to BPH. Cardiovasc Intervent Radiol. 2017;40(10):1490-500.

4. Abt D, Hechelhammer L, Mullhaupt G, Markart S, Gusewell S, Kessler TM, et al. Comparison of prostatic artery embolisation (PAE) versus transurethral resection of the prostate (TURP) for benign prostatic hyperplasia: randomised, open label, non-inferiority trial. BMJ. 2018;361:k2338.

5. Pisco J, Campos Pinheiro L, Bilhim T, Duarte M, Rio Tinto H, Fernandes L, et al. Prostatic arterial embolization for benign prostatic hyperplasia: short- and intermediateterm results. Radiology. 2013;266(2):668-77.

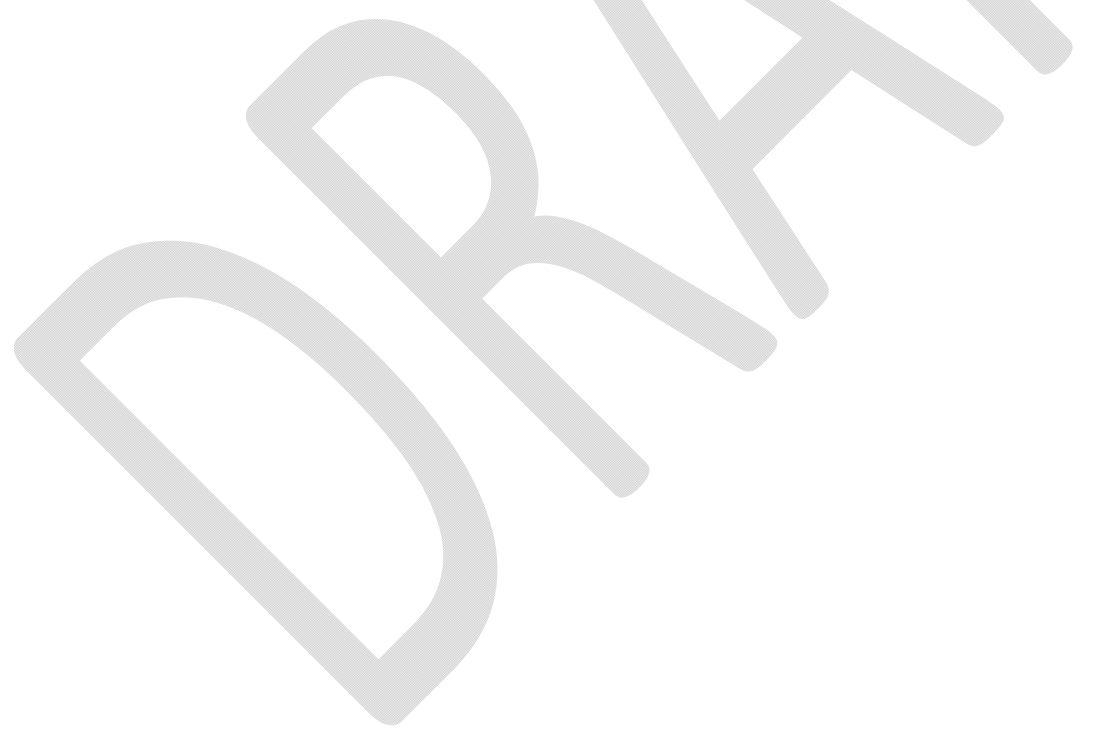




\section{Figures and . s}

Fig. 1. Procedural images. (A) Left-sided oblique view showing contrast injection in the left prostatic artery (LPA) with progression into a left arterial shunt (LS) leading to the left dorsal penile artery (LDPA). (B) Left-sided oblique view showing coiling material applied to the left arterial shunt. (C) Right-sided oblique view showing a narrow, filiform right arterial shunt (RS) connecting the right prostatic artery to the right dorsal penile artery (RDPA); the contralateral coil is also visible.

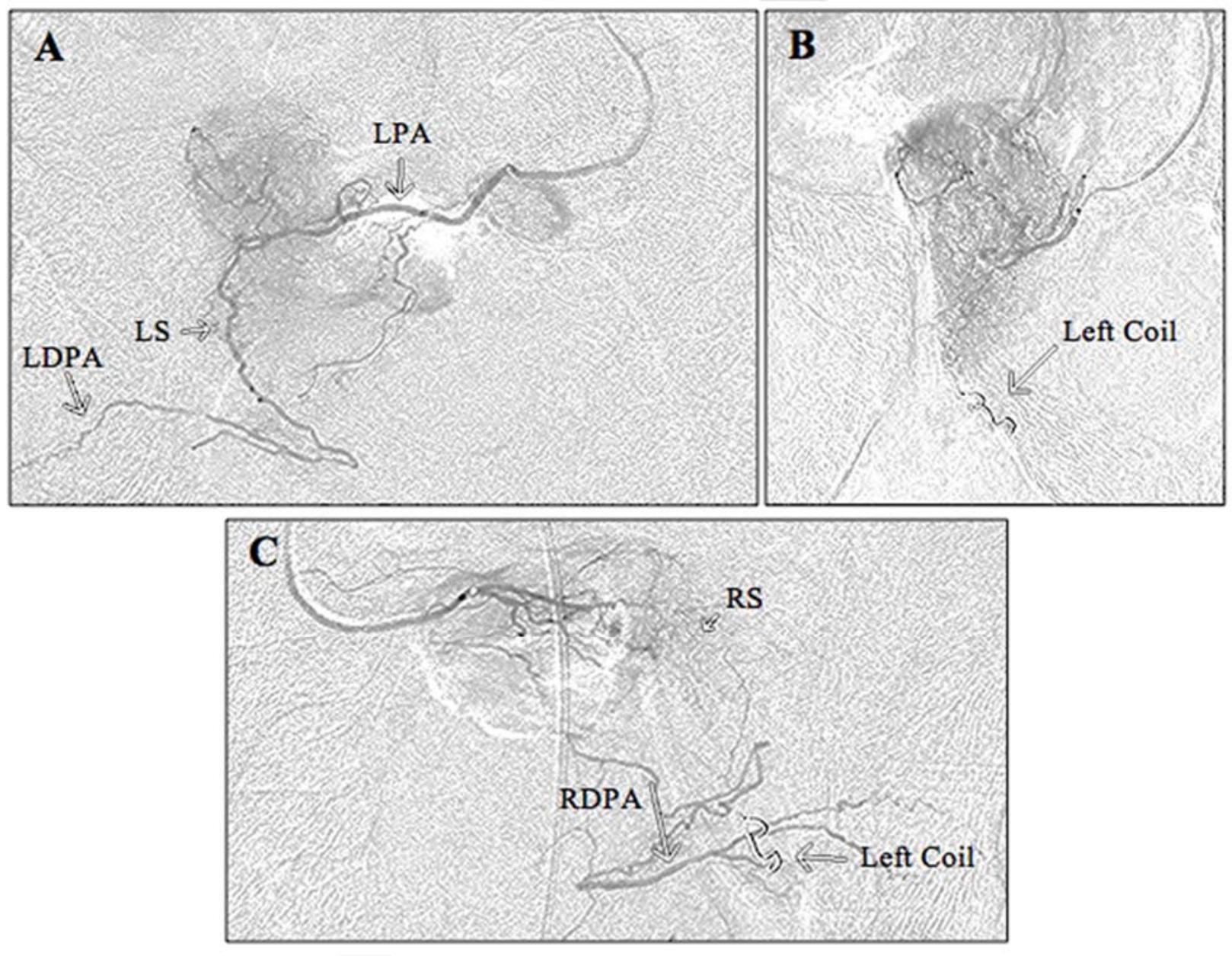


Fig. 2. Clinical photographs. (A) The patient presented to the emergency room 10 days following the procedure with a painful, dark lesion located on the penile glans. Physical examination revealed a necrotic, 1-cm ulcer surrounded by a zone of erythema just adjacent to the right side of the uretral meatus. (B) Picture taken 7 months after the procedure showing complete resolution of the penile lesion, which had completely healed after about 5 weeks.

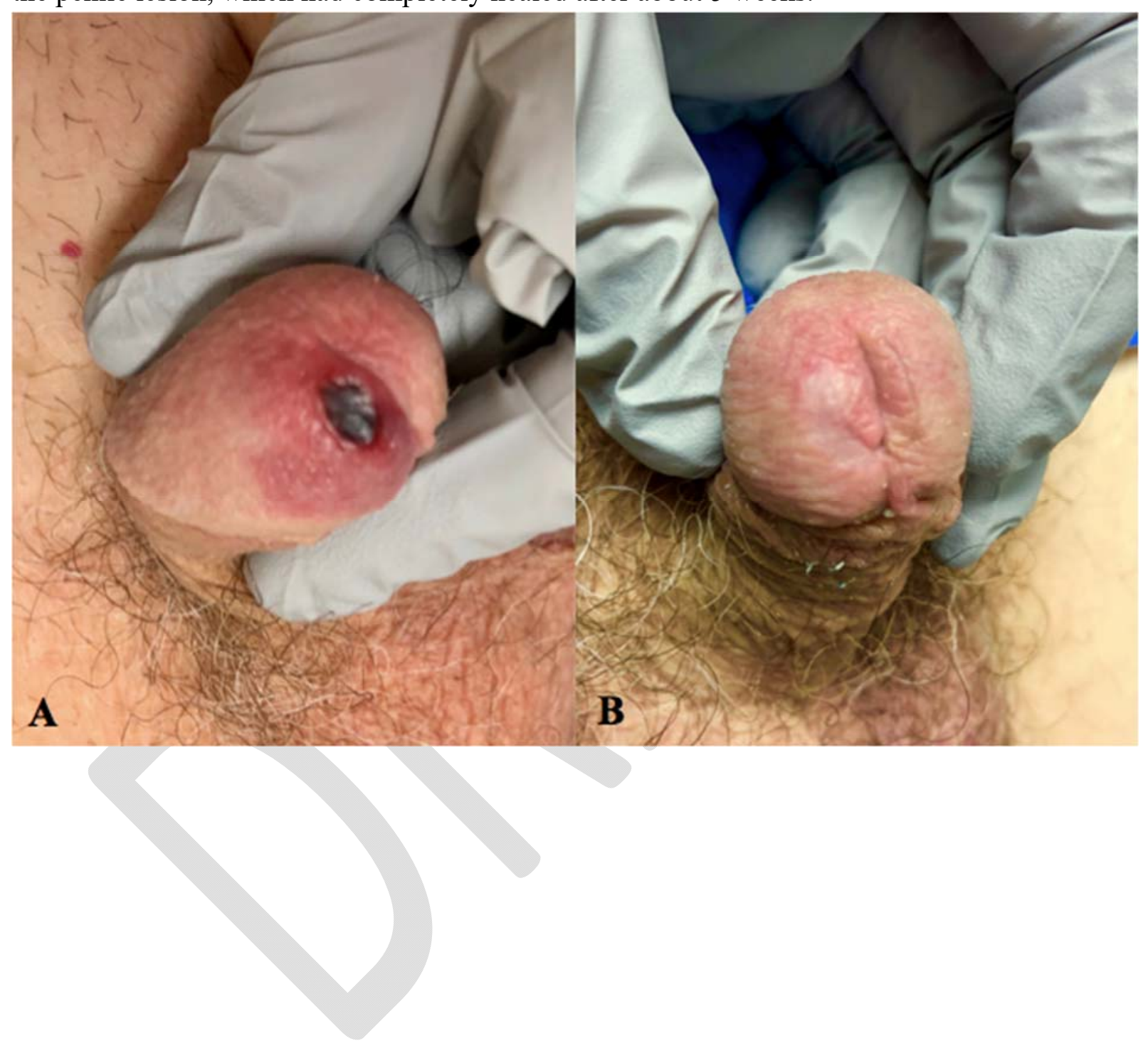

